\title{
TEMPO DE VIDA ÚTIL DA REPRESA VELHA DA MICROBACIA DO CÓRREGO DA BARRINHA, PIRASSUNUNGA, SP, BRASIL
}

\author{
A.G.M. Bufon ${ }^{1}$, S.M. Tauk-Tornisielo ${ }^{1}$, A.C.S. Pião ${ }^{2}$ \\ ${ }^{1}$ Universidade Estadual Paulista, Centro de Estudos Ambientais, Av. 24-A, 1515, CEP 13506-900, Rio Claro, \\ SP, Brasil. E-mail: seb@rc.unesp.br
}

\section{RESUMO}

\begin{abstract}
Conservação das águas continentais torna-se cada vez mais necessária devido aos crescentes impactos ambientais negativos e ao aumento acelerado da sua escassez. No Centro de Pesquisa e Gestão de Recursos Pesqueiros Continentais (CEPTA/IBAMA), no Município de Pirassununga, SP, localiza-se a represa Velha. O tempo de vida útil desta represa foi determinado por meio da taxa de sedimentação, comparando-se duas metodologias distintas. Uma delas consistiu na batimetria com guincho hidrométrico e a outra utilizando câmaras de sedimentação. As coletas das amostras nestas últimas ocorreram na coluna de água a $50 \mathrm{~cm}$ acima do sedimento, nos períodos de seca e chuvoso, durante doze meses. Com esta metodologia pode-se calcular a taxa de sedimento de 7,0 mg.cm ${ }^{-2}$ dia ${ }^{-1}$ e a densidade anual de $0,66 \mathrm{mg} . \mathrm{cm}^{-3}$. O cálculo do tempo de vida útil para a represa Velha foi de 7,5 anos. Por meio da batimetria foi determinada a perda da profundidade ao longo de toda a extensão desta represa, sendo em média, de 0,07 m.ano-1. Concluiu-se que serão necessários 72 anos para que esta represa possa ser totalmente assoreada, este tempo podendo ser reduzido se não houver ações mitigadoras dos impactos atuais e planejamento para evitar outros que possivelmente poderão ocorrer.
\end{abstract}

PALAVRAS-CHAVE: Represa, taxa de sedimentação, batimetria, impacto ambiental.

\section{ABSTRACT}

TIME LIFE THE “VELHA” DAM BASINS OF THE STREAM BARRINHA, PIRASSUNUNGA, SÃO PAULO STATE, BRAZIL. Conservation of continental waters is becoming increasingly necessary due to the growing negative environmental impacts and the acceleration of its scarcity. In the Center of Research and Management of Continental Fishing Resources (CEPTA/IBAMA), city of Pirassununga, SP, Brazil, located in the "Velha" dam. The lifespan of this dam was determined by sedimentation rate, comparing two different methodologies. One was the bathymetry with a hydrometric and other hydrometric using sedimentation chambers. The samples collections in these last ones had occurred in the water column the $50 \mathrm{~cm}$ above the sediment, in the dry and rainy periods, during twelve months. With this methodology can be calculated the sediment rate of $7.0 \mathrm{mg} \cdot \mathrm{cm}^{-2} \mathrm{day}^{-1}$ and $0.66 \mathrm{mg} \cdot \mathrm{cm}^{-3}$ annual density. The calculation of lifetime for the "Velha" dam was 7.5 years. Through the bathymetry was determined the loss of depth along the entire length of this dam, with an average, of 0.07 m.year-1. It was concluded it will take 72 years for this dam to be completely silted up, this time can be reduced if no mitigating actions for the current impacts and planning to avoid others who may possibly occur.

KEY WORDS: Dam, sedimentation rate, bathymetry, environmental impact.

\section{INTRODUÇÃO}

A disponibilidade de água e sua qualidade podem limitar o desenvolvimento econômico de um país. Dentro do território nacional, portanto, devem-se prevenir as perdas hídricas e providen- ciar mecanismos e ações para economia e reuso da água nos sistemas de distribuição. Tanto qualidade e quantidade da água são consequências do uso e ocupação do solo, da expansão demográfica, do desmatamento e das mudanças globais climáticas. A gestão integrada dos recursos hídricos juntamente

${ }^{2}$ Universidade Estadual Paulista, Instituto de Geociências e Ciências Exatas, Departamento de Estatística, Matemática Aplicada e Computação, Rio Caro, SP, Brasil. 
com a gestão comunitária da água podem minimizar e desacelerar o grau de poluição hoje existente nos corpos de água (Tundisi, 2000).

Atualmente, grandes represas têm sido construídas para diversos fins, tais como: abastecimento de água, fonte de energia, irrigação e recreação. Porém, a partir da construção desses reservatórios, surgem os grandes problemas de assoreamento, devido a não conservação dos solos a montante dos rios contribuintes, além dos problemas decorrentes da expansão de áreas urbanas, provocando a diminuição da sua vida útil. Os reservatórios da América do Sul e, em especial, do Brasil, são importantes ecossistemas dentro da rede hidrográfica, requerendo gerenciamentos complexos. São considerados coletores de eventos ao longo da bacia hidrográfica onde foram construídos (TundIII, 1988; TundISI; STRAŠKRABA, 1994). Dos vários problemas observados nos reservatórios, destacam-se a deposição de materiais advindos das atividades agrícolas, do uso inadequado do solo e dos efluentes industriais e domésticos. Os problemas de transporte de material alóctone e sua deposição podemagravar o estado do reservatório, tendo como consequência final o acúmulo de compostos tóxicos, diminuição do oxigênio e o aumento da turbidez.

Apesar da proposta da análise de determinados fatores que atuam na caracterização de possível sedimentação dos reservatórios: vazão, tempo de residência da água e a seção transversal dos rios formadores dos reservatórios; características morfométricas e a localização e uso da bacia hidrográfica, os maiores problemas nos reservatórios relacionam-se com os acúmulos de materiais particulados trazidos na época de chuva, além do basalto, de alumínio, carbonato dissolvido e outros tipos de minerais (KRUSCHE; MozETo, 1999). O aumento da deposição demateriais interfere nos processos físicos, químicos e microbiológicos e na própria perda da capacidade de armazenamento da água. O transporte e a deposição de sedimentos são processos dominantes em reservatórios, influenciando de forma significativa à resposta ecológica do sistema. O sedimento pode ser um dos maiores veículos de poluição da água por peso e volume, sendo também o grande transportador e catalisador de defensivos agrícolas, resíduos orgânicos, nutrientes e organismos patogênicos (THORNTON, 1990). É necessário conhecer o processo de sedimentação em reservatórios, para se estabelecer seu gerenciamento adequado. O padrão desse processo e a variação entre as taxas de acumulação do sedimento são ferramentas importantes para prevenir a perda da capacidade de armazenamento (HenRY; MARICATO, 1996).

O objetivo do presente estudo foi determinar o tempo de vida útil da represa Velha, por meio da taxa de sedimentação utilizando-se duas metodologias distintas. Estas consistiram na batimetria da represa e a utilização de câmaras de sedimentação, que geram informações que poderão subsidiar os estudos referentes às represas.

\section{MATERIAL E MÉTODOS}

\section{Área de estudo}

Aárea deestudolocaliza-segeograficamenteem $21^{\circ}$ $55^{\prime} 35^{\prime \prime}$ S e $47^{\circ} 22^{\prime} 00^{\prime \prime} \mathrm{W}$, e $21^{\circ} 57^{\prime} 30^{\prime \prime}$ S e $47^{\circ} 24^{\prime} 00^{\prime \prime} \mathrm{W}$, aproximadamente a $575 \mathrm{~m}$ acima do nível do mar, no Município dePirassununga, SP, no Centro dePesquisa eGestãodeRecursosPesqueiros Continentais(CEPTA), do Instituto Brasileiro do Meio Ambiente e dos Recursos Naturais (IBAMA). Neste centro existe a represa Velha que é abastecida pela microbacia do Córrego da Barrinha, com uma área de aproximadamente 862 ha, que faz parte da bacia do Rio Mogi-Guaçu. A maior parte da área desta microbacia, aproximadamente 259 ha $(40 \%)$, pertenceaoCEPTA/IBAMA ondeépraticada a aquicultura (KLEEREKOPER, 1941).

A microbacia em estudo é dividida em nove pequenas propriedades, entre elas a já citada anteriormente, as quais desenvolvem diversas atividades agropecuárias, como a plantação de citros $(13,4 \%)$, cana-de-açúcar $(15,1 \%)$, culturas temporárias $(18,0 \%)$, criação de gado bovino $(5,1 \%)$, tanques de piscicultura $(0,9 \%)$, além de mineração $(0,2 \%)$, reflorestamento $(0,3 \%)$, depósito de lixo $(0,1 \%)$ e área de empréstimo $(0,3 \%)$, totalizando $53,4 \%$. As áreas remanescentes de cobertura florestal incluem um parque ecológico municipal $(0,6 \%)$, cerrado típico $(6,4 \%)$, capoeira $(7,4 \%)$, cerradão $(8,1 \%)$, represas, lagos e rio (1,1\%) e várzeas (5,1\%), totalizando $28,7 \%$, dos quais aproximadamente $21,0 \%$ encontram-se na área do CEPTA. Além desses usos, há uma forte influência demográfica nessa microbacia, pois esta área é recortada por estrada asfaltada (3,7\%) e limitada por dois distritos urbanos (Cachoeira de Emas e Vila Santa Fé) (14,3\%), sofrendo diretamente os efeitos da pressão antrópica (BUFON, 2002).

O coeficiente de escoamento superficial ("runo$\mathrm{ff}^{\prime \prime}$ ) da represa "Velha" éigual a 0,40 mm, em função do tipo de solo, da cobertura vegetal e da declividade. A intensidade de chuva de determinado tempo de recorrência e de duração igual ao tempo de concentração da bacia é de $72 \mathrm{~mm} . \mathrm{h}^{-1}$ (KLEEREKOPER, 1941).

A represa Velha foi construída no final de 1939 para armazenar água, principalmente para o abastecimento de tanques, viveiros e laboratórios do CEPTA/IBAMA. Em 1940 seu volume era de 95.240,00 $\mathrm{m}^{3}$. No entorno dessa represa ao Sul, Leste ocorre mata secundária (resquício de Mata Atlântica) e ao Norte há uma barragem de $300 \mathrm{~m}$ de comprimento, construída de terra, com uma plantação de bambu e uma área molhada aproximada de 5 ha. Durante 
algum tempo foram realizadas algumas melhorias da represa, sendo que no ano de 1998, o volume útil dela era de $97.960,70 \mathrm{~m}^{3}$ e em 1999, de 96.704,17 $\mathrm{m}^{3}$ (KleEReKoper, 1941; Bufon, 1999). O volume morto em um ano foi de $1.256,53 \mathrm{~m}^{3}$. A profundidade média da represa "Velha" é de 1,25 m (Bufon, 1999). O único afluente dessa represa é o córrego da Barrinha.

Para as análises das variáveis, as amostras foram coletadas mensalmente, nos períodos seco/frio (junho, julhoeagosto de 2000 eabril de2001) echuvoso/quente (outubro e dezembro de 2000, janeiro e fevereiro de 2001). Em cada um deles foi instalada verticalmente uma câmara de sedimentação, com altura de $70 \%$ da profundidade total, segundo LeITE (1998). (Fig. 1).

Para avaliar a deposição de material em suspensão na represa, foram utilizadas, como já mencionado, câmaras de sedimentação confeccionadas de tubos de PVC de $10 \mathrm{~cm}$ de diâmetro por $30 \mathrm{~cm}$ de comprimento, o que representa uma proporção aproximada de comprimento/diâmetro de 3:1 (MARICATO, 1994) (Fig. 2). Os pontos onde foram colocadas as câmaras de sedimentação individualmente tiveram as seguintes profundidades: ponto 1, 1,39 m; ponto 2, 0,73 m; ponto 3, 0,99 m; ponto 4, 4,40 m; ponto 5,2,93 $\mathrm{m}$ e o ponto $6,2,08 \mathrm{~m}$. As amostras de sedimentação foram coletadas conforme a metodologia usada por
Maricato (1994) e LeITE (1998), com periodicidade mensal nos períodos seco echuvoso, predominantes na área de estudo.

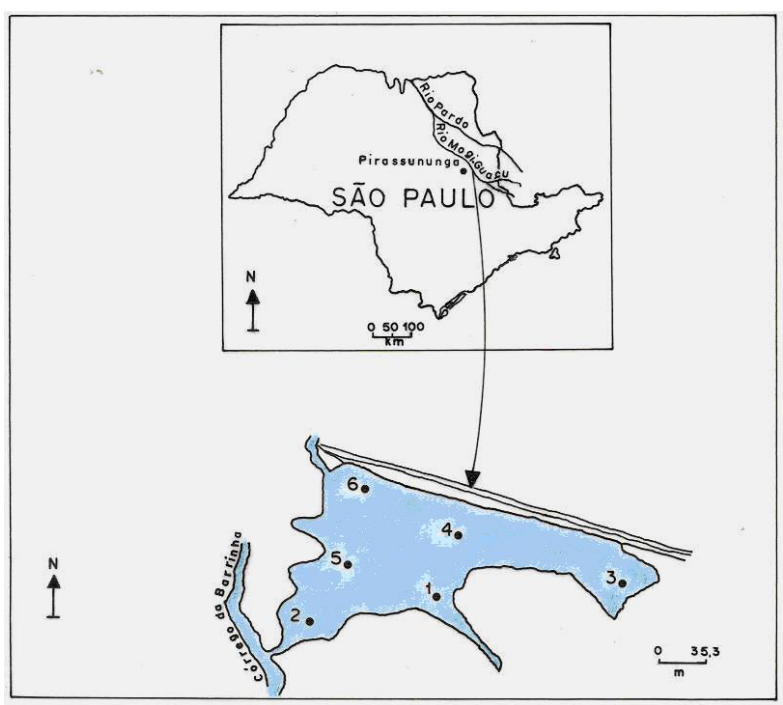

Fig. 1 - Croqui da represa Velha mostrando os pontos de amostragens. Fonte: Bufon (2002). Legenda: Ponto 1 antigo cercado da represa, ponto 2 - entrada do córrego da Barrinha dentro da represa, ponto 3 - antigo banco de macrófitas, ponto 4 - barragem, ponto 5 - meio da represa, ponto 6 - antigo local dos tanques-rede.

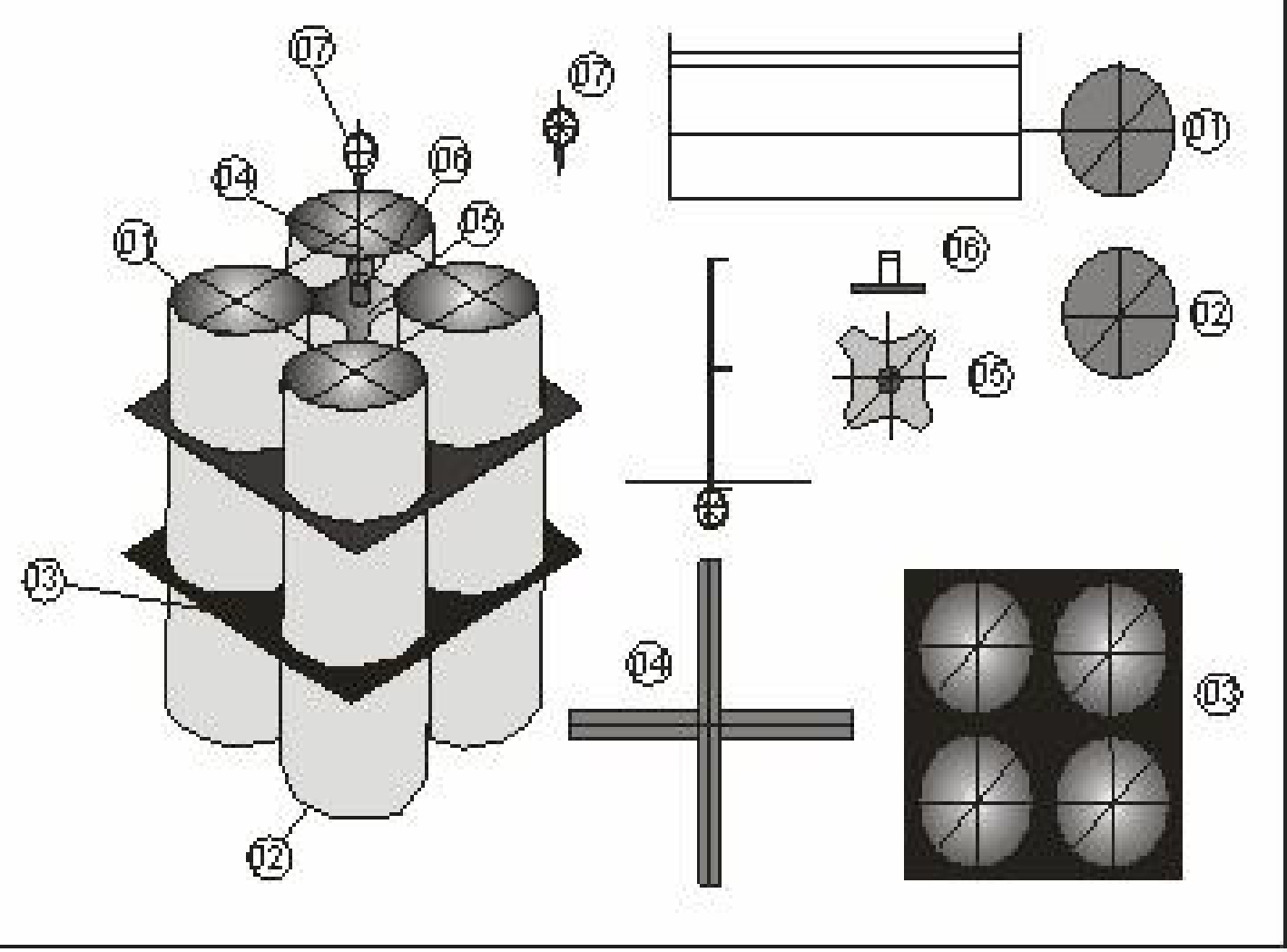

Fig. 2 - Esquema das câmaras de sedimentação. 1- Abertura da câmara; 2- fundo da câmara; 3 - suporte das câmaras; 4 - Rosca de trava; 5 - Trava das câmaras; 6 - Parafuso da trava das câmaras; 7 - Alça de amarração. Fonte: BuFON (2002). 
Antes da colocação, as câmaras foram preenchidas com água destilada, evitando-se assim a deposição de material antes do início do experimento. Posteriormente, elas foram colocadas nas profundidades estabelecidas, sendo amarradas a um flutuador de madeira com boias, o qual foi ancorado em cada ponto de amostragem, permitindo, desta forma, avaliar a diferença espacial na sedimentação do material produzido, bem como as contribuições do principal tributário. Após o período de incubação, os conteúdos das câmaras foram recolhidos, homogeneizados e colocados em galões e levados ao laboratório.

No laboratório, um volume conhecido de água foi filtrado em membranas do tipo Whatman GF/C, determinado em amostras brutas e fracionadas (malhas de 68 e $20 \mu \mathrm{m}$ ), retido em filtros de fibra de vidro, previamente calcinados e de massa conhecida (M1), por técnica gravimétrica (WETZEL; LIKENS, 1991). Os filtros contendo material particulado foram secos em estufa a $60^{\circ} \mathrm{C}$ durante um período de $24 \mathrm{~h}$, resfriados e pesados (M2). Após, foram calcinados em mufla a $480^{\circ} \mathrm{C}$, por $60 \mathrm{~min}$, resfriados e pesados (M3). As diferenças de massas entre: M1 e M2; M1 e M3; M2 e M3 forneceram as massas dos materiais em suspensão total, inorgânico e orgânico, respectivamente.

A taxa de sedimentação do material particulado foi correspondente à quantidade de material da amostra filtrada, corrigida para o volume médio das câmaras de sedimentação, expressos em unidades de área $\left(\mathrm{cm}^{2}\right)$ e de tempo (número de dias do período de incubação). A taxa de sedimentação foi determinada pela seguinte fórmula: T.S. $=\mathrm{Vc}^{\prime \prime \prime} \mathrm{C} / \mathrm{Ac}^{\prime \prime \prime} \mathrm{T}$; onde: $\mathrm{Vc}$ = volume das câmaras de sedimentação $(2,36 \mathrm{~L}) ; \mathrm{C}=$ concentração de material em suspensão dentro das câmaras (mg/L); Ac = área da abertura da superfície da câmara de sedimentação $\left(78,54 \mathrm{~cm}^{2}\right)$; $\mathrm{e} \mathrm{T}=$ tempo (dias) (MARICATO, 1994).

As batimetrias da represa foram realizadas no mês de agosto de 2000 e de 2001, nos pontos com distâncias entre si de $10 \mathrm{~m}$, totalizando 389 pontos. Estas foram determinadas com um guincho hidrométrico (fabricação nacional), tendo cabos de aço para 15 e $25 \mathrm{~m}$ de comprimento e um contador digital para indicar continuamente a extensão do cabo desenrolado com precisão de centímetro (CARVALHO, 1994). Utilizou-se a média das profundidades dos pontos de coletas para o cálculo de vida útil da represa Velha realizado com a seguinte equação: $\mathrm{Tv}=\mathrm{V}^{\prime \prime \prime} \mathrm{T} / \mathrm{S}$; onde: $\mathrm{Tv}=$ tempo de vida útil do reservatório; $\mathrm{V}=$ volume do reservatório; $\mathrm{S}=$ material depositado em anos; e T = tempo de comparação entre uma medida e outra (Bufon, 1999). A batimetria consiste, basicamente, no conhecimento da profundidade do leito do rio e da represa, utilizando-se um referencial, como RN (referência de nível). Esta ferramenta possibilita o estudo do acompanhamento temporal do material depositado nos corpos de água superficiais.

\section{RESULTADOS E DISCUSSÃO}

Os estudos aqui realizados mensalmente com as câmaras de sedimentação, durante o período de $24 \mathrm{~h}$, demonstraram que o valor médio mensal da taxa de sedimentação, durante um ano, foi de $7,0 \mathrm{mg} \cdot \mathrm{cm}^{-2} \mathrm{dia}^{-1}$ e a densidade anual de $0,659 \mathrm{mg} \cdot \mathrm{cm}^{-3}$. O cálculo do tempo de vida útil para a represa Velha foi de 7,5 (sete e meio) anos, ou seja, para que a mesma seja totalmente assoreada.

Em estudos paralelos a este (BuFON; LANDIN, 2007), por meio da análise de agrupamento, foi verificado que os valores de radicais fosfatos e fósforo total se relacionaram diretamente com o material de suspensão nas águas da represa aqui em estudo, no período seco e ele não foi observado no período chuvoso. A análise estatística utilizada permitiu concluir que a represa Velha, no Município de Pirassununga, vem apresentando uma estratificação térmica e pode ser considerada oligotrófica.

Considerando-se qualitativa e quantitativamente os fatores abióticos e bióticos presentes na represa Velha (Bufon, 2002), pode-se fazer uma previsão, por meio das batimetrias realizadas, de 72 (setenta e dois) anos o tempo no qual esta represa deverá estar totalmente assoreada. Este valor está relacionado com a perda obtida da profundidade máxima e mínima que oscilou entre 0,10 a 5,0 m na represa Velha (Fig. 3). As análises estatísticas demonstraram a ocorrência da perda média de 0,07 $\mathrm{m}$ de profundidade ao longo de toda a extensão da represa.

De acordo com as diferentes metodologias usadas por autores distintos, podem-se verificar algumas defasagens do valor de vida útil de um mesmo reservatório, considerando-se também os períodos nos quais os estudos foram realizados. Como pode ser observado quando se comparam os resultados obtidos por Coelho (1993) e os de Leite (1998). O uso e ocupação do solo, atividades antrópicas e outros fatores contribuem para que o meio seja dinâmico, o que interfere nos valores de vida útil de reservatórios. Um fator importante observado a campo, que está relacionado ao processo de assoreamento, é a retirada da vegetação natural nas margens do reservatório. A preservação e a manutenção das matas ciliares tornam-se barreiras ao carreamento para o reservatório, por meio das enxurradas, dos detritos sólidos erodidos e aumentam a resistência dos solos das margens à erosão provocada pelo embate das ondas. 


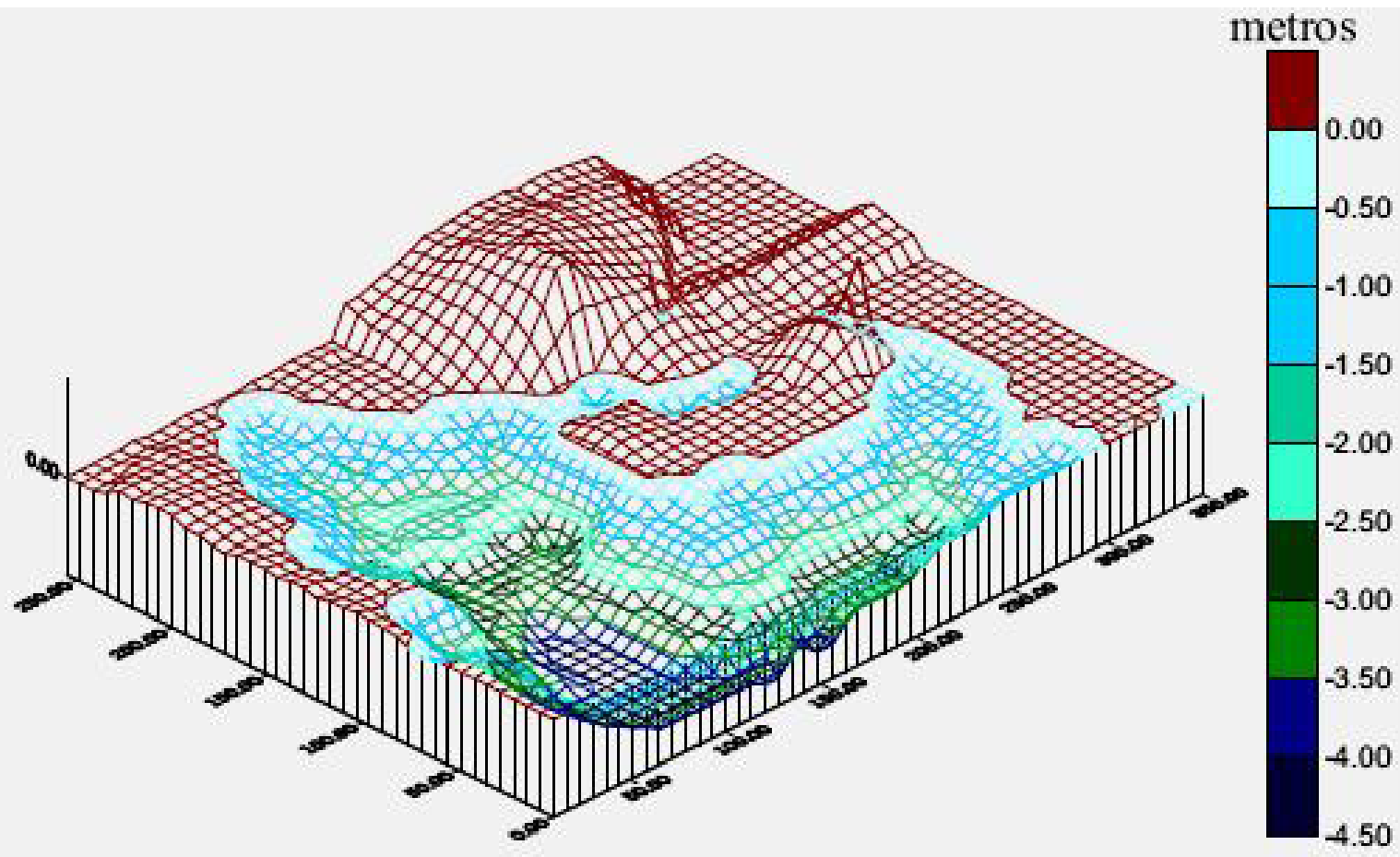

metros

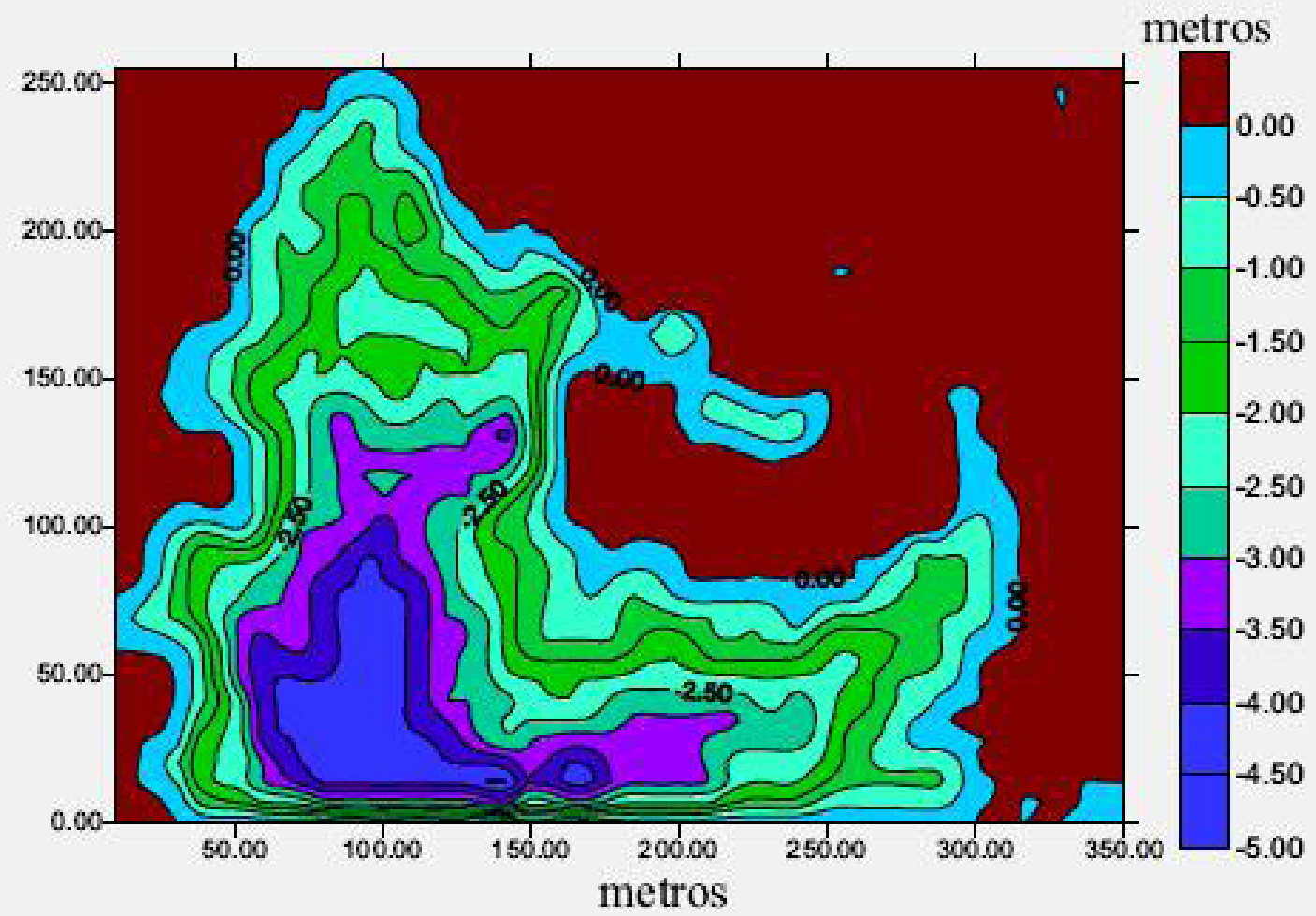

Fig. 3 - Planta batimétrica das curvas de nível e em 3D [terceira dimensão da represa Velha (CEPTA/IBAMA/SP)]. Fonte: BuFON (2002).

Villela; PONCE (1986) citaram os fatores que influenciam no depósito de sedimentos e a sua dis- tribuição no reservatório, na operação, no sedimento transportado, no tamanho do reservatório, na decli- 
vidade do vale do rio, na vegetação e no deflúvio do efluente. Esses resultados, juntamente com estes aqui encontrados são, certamente, oriundos dos problemas ambientais, locais ou regionais, e poderão subsidiar o gerenciamento eficaz da microbacia em estudo, particularmente da represa. Este gerenciamento correto será, certamente, uma atividade de longo prazo que deverá ser encaixada no processo de desenvolvimento integrado da área em pauta. Evidentemente os fatores abióticos e bióticos não são constantes devido à aceleração da degradação ambiental, decorrente do uso e ocupação do solo ao redor da represa. Assim, o tempo de 72 (setenta e dois) anos previstos poderá ser modificado, caso haja um planejamento e gerenciamento ambiental satisfatórios. Pela comparação dos dados topográficos da época da construção do reservatório da usina de Cachoeira Dourada com os dados batimétricos medidos por alguns autores, houve perda de $38,5 \%$ da capacidade de armazenamento original. A expectativa de vida útil da represa foi estimada, nessa pesquisa, a continuar o atual ritmo de assoreamento de aproximadamente 145 anos (CABRAL et al., 2008).

\section{CONCLUSÕES}

Conclui-se que os resultados obtidos indicam que deverão ser repor meio através de reflorestamento das áreas de preservação permanente ao longo de todo o Córrego da Barrinha; manejo mais adequado do uso e ocupação do solo, com construções de curvas de níveis e desassoreamento da represa Velha. Também verificou-se que a técnica com câmaras de sedimentação não é adequada para o cálculo de vida útil de uma represa, com as mesmas características desta estudada.

Um monitoramento evidentemente necessário ficou evidenciado com o levantamento batimétrico; assim outras batimetrias deverão ser realizadas com frequência adequada. $\mathrm{O}$ atual levantamento batimétrico forneceu a situação atual do assoreamento e poderá re-orientar o plano de gestão desta microbacia aqui estudada.

\section{AGRADECIMENTOS}

Ao Centro de Pesquisa e Gestão de Recursos Pesqueiros Continentais - CEPTA/IBAMA, pelo apoio e estrutura utilizada neste trabalho; ao Centro de Estudos Ambientais - CEA da Universidade Estadual Paulista (UNESP), Campus de Rio Claro - SP, pela atenção dedicada dos docentes e técnicos e à Fundação de Amparo à Pesquisa do Estado de São Paulo - FAPESP pela concessão da bolsa de Mestrado e auxílio à pesquisa, o qual foi de grande importância para execução deste trabalho (Processo 00/05364-0).

\section{REFERÊNCIAS}

BUFON, A.G.M. Variação da taxa de sedimentação na represa Velha (CEPTA/IBAMA/SP) e sua influência sobre as carac-terísticas limnológicas do sistema. Um estudo de impacto ambiental, Pirassununga/SP. 1999. $80 \mathrm{f}$. Monografia (Graduação em Engenharia de Agrimensura) - Faculdade de Engenharia de Agrimensura de Pirassununga, Pirassununga,1999.

BUFON, A.G.M. Variação temporal e espacial da taxa de sedimentação e das características limnológicas na microbacia do córrego da Barrinha, no município de Pirassununga, SP. 2002. 180 f. Dissertação (Mestrado em Conservação e Manejo de Recursos) - Centro de Estudos Ambientais, Universidade Estadual Paulista, Rio Claro, 2002.

BUFON, A.G.M.; LANDIN, P.M.B. Analise da qualidade da água por metodologia estatística multivariada na represa Velha (CEPTA/IBAMA/Pirassununga/ SP). Holos Environment, v.7, n.1, p.42-59, 2007.

CABRAL, J.B.P.; FERNANDES, L.A.; SCOPEL, I.; BECEGATO, V.A.; FIORI, A.P. valiação do estado de assoreamento do reservatório de Cachoeira Dourada (GO/MG). Sociedade \& Natureza, 2008. Disponível em: <http://www. 10.1590/S1982-45132009000100007>. Acesso em: 29 out. 2009.

CARVALHO, N.O. Hidrossedimentologia prática. Rio de Janeiro: CPRM, 1994. 372p.

COELHO, C.P. Análise do processo de assoreamento do reservatório de Americana - SP. 1993. 69 f. Dissertação (Mestrado em Geociências e Meio Ambiente) - Instituto de Geociências e Ciências Exatas, Universidade Estadual Paulista, Rio Claro, 1993.

HENRY, R; MARICATO, F.E. Sedimentation rates of tripton in Jurumirim reservoir (São Paulo, Brazil). Limnologica, v.26, p.15-25, 1996

KLEEREKOPER, H. Estudo limnológico da bacia do rio Mogi-Guaçu: observações sobre a represa da Estação Experimental de Caça e Pesca do Ministério da Agricultura em Emas, SP. Rio de Janeiro: Serviço de Informação Agrícola, 1941.

KRUSCHE, A.V.; MOZETO, A.A. Seasonal variations in water quality of an oxbow lake in response to multiple short-time pulses of flooding (Jataí Ecological Sation - Mogi-Guaçu River, Luiz Antonio, SP- Brazil). Anais da Academia Brasileira de Ciências, v.71, n.4, p.777789, 1999. 
LEITE, M.A. Variação espacial e temporal da taxa de sedimentação no Reservatório de Salto Grande (SP) e sua influência sobre as características limnológicas do sistema. 1998. 170 f. Dissertação (Mestrado em Ciências da Engenharia Ambiental) - Escola de Engenharia de São Carlos, Universidade de São Paulo, São Carlos, 1998.

LOPEZ, S.J.L. Mathematical modeling of sediment deposition in reservoir. Colorado: Colorado State University, 1978. (Hydrology Papers).

MARICATO, F.E. Caracterização físico-química e sedimentação nas regiões de desembocadura dos rios Paranapanema e Taquari, e no reservatório de Jurumirim (Alto Paranapanema - SP). 1994. 205 f. Dissertação (Mestrado em Ciências da Engenharia Ambiental) - Escola de Engenharia de São Carlos, Universidade de São Paulo, São Carlos, 1994.

STRAŠKRABA, M. Limnological basis for modeling reservoir ecosystem. In: ACKERMANN, W.C.; WHITE, G.F.; WORTHINGTON, E.B. (Ed.). Man made lakes: their problems and environment effects. Washington, DC: American Geophysical Union, 1973. p.517-535. (Geophysical Union Graphs, 17).

THORNTON, K.W. Sedimentary process. In: THORNTON, K.W.; KIMMEL, B.L.; PAYNE, F.E. (Ed.). Reservoir limnology: ecological perspectives. New York: John Wiley Sons, 1990. p.43-69.
TUNDISI, J.G. (Ed.). Limnologia e manejo de represas. São Carlos: EESC/USP; CRHEA; ACIESP, 1988. v.1, t.1, 506p. (Monografias em Limnologia).

TUNDISI, J.G. Diretrizes para o gerenciamento de lagos. São Carlos: Instituto Internacional de Ecologia, 2000. v.1, 184p.

TUNDISI, J.G.; STRAŠKRABA, M. Ecological basis for the application of ecotechnologies to watershed/ reservoir and management. Acta Limnologica Brasileira, v.5, n.1, p.49-72, 1994.

VALERA, N.E.; IZQUIERDO, G.C. Problemas y soluciones de sedimentación en la planta del bajo Anchicaya. In: SEMINARIO LATINOAMERICANO SOBRE PRESAS Y EMBALSES, 1, Bogotá. Anais... Bogotá, 1984.

VILLELA, S.M.; PONCE, R.J.C. Assoreamento de reservatórios. Boletim de Hidráulica e Saneamento, v.1, n.8, p.1 1-30, 1986.

WETZEL, R.G; LIKENS, G.E. Limnological analysis. New York: Springer Verlag, 1991. 391p.

Recebido em 15/10/07

Aceito em 29/10/09 